\title{
Densification-induced conductivity percolation in high-porosity pharmaceutical microcrystalline cellulose compacts
}

\author{
M. Strømme a) and G. A. Niklasson \\ Department of Materials Science, The Angström Laboratory, Uppsala University, Box 534, \\ SE 75121 Uppsala, Sweden \\ R. Ek \\ Department of Pharmacy, The Biomedical Centre, Uppsala University, Box 580, SE 75123 Uppsala, Sweden
}

(Received 4 September 2002; accepted 3 December 2002)

\begin{abstract}
The percolation theory is established as a useful tool in the field of pharmaceutical materials science. It is shown that percolation theory, developed for analyzing insulator-conductor transitions, can be applied to describe imperfect dc conduction in pharmaceutical microcrystalline cellulose during densification. The system, in fact, exactly reproduces the values of the percolation threshold and exponent estimated for a three-dimensional random continuum. Our data clearly show a crossover from a power-law percolation theory region to a linear effective medium theory region at a cellulose porosity of $\sim 0.7$. (C) 2003 American Institute of Physics. [DOI: 10.1063/1.1539902]
\end{abstract}

Percolation theory has been widely used to model physical phenomena in disordered structures. ${ }^{1,2}$ The theory has been applied not only in physics but also in various other fields such as chemistry, biology, and geology. ${ }^{3,4}$ In particular, the physics of porous materials has benefited greatly from percolation concepts. In this letter, we establish percolation theory as a useful tool also in the field of pharmaceutical materials science.

Given a random mixture of conducting and insulating materials, one of the most striking predictions of the percolation theory is the existence of the so-called percolation threshold. As the volume fraction $f$ of the conducting component increases, the percolation threshold signifies the volume fraction $f_{c}$, at which the composite abruptly becomes conducting. Close to the percolation threshold, electrical properties of the composite, such as the dc conductivity, exhibit scaling relations. A review of the scaling theory for electrical properties can be found, for example, in Ref. 5. Specifically above $f_{c}$, the dc conductivity is predicted to vary as

$$
\sigma_{\mathrm{dc}}=\sigma_{0}\left(f-f_{c}\right)^{t},
$$

where $\sigma_{0}$ is a constant proportional to the conductivity of the conducting material, and $t$ is the scaling exponent. This type of equation applies to percolation phenomena, in general, and not only to the conductivity, but the magnitude of $f_{c}$ and $t$ may however depend on the physical property under consideration.

Equation (1) is expected to hold in a limited critical region close to $f_{c}$. Outside of this region, a crossover to the behavior obtained from mean field- or effective medium theory is expected. Such a crossover was first observed in numerical simulations of random resistor networks by Kirkpatrick. ${ }^{6}$ It was shown that the scaling region where Eq. (1) is obeyed can be substantial; in the case studied in Ref. 6, it extended beyond $f-f_{c} \sim 0.2$.

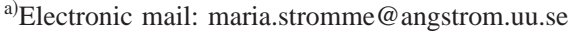

In a three-dimensional (3D) system where insulator particles and conducting particles are randomly mixed, a percolation threshold $f_{c}$ at a volume fraction of $15 \%$ conducting particles is predicted by considering packing in different types of lattices. ${ }^{7}$ By random resistor simulations, the exponent of such a system is expected to be very close to 2.0. ${ }^{8}$ However, values of critical metal volume fractions at the insulator-metal transition have been reported from $15 \%$ to $60 \%$ for different composite materials. ${ }^{9}$ These disparate values have later been explained by differences in material structure, random or granular, and in film dimensionality. ${ }^{1}$ Whereas a perfect $3 \mathrm{D}$ random continuum has $f_{c}=0.15$, a two-dimensional randomly mixed system has $f_{c}=0.50$. A granular structure, i.e., a structure where conducting grains do not agglomerate to form large clusters, but instead appear to remain separate and surrounded by very thin layers of insulator, increases these values. ${ }^{1,10}$ Hence, percolation theory has been recognized as an excellent tool to predict cluster geometry and statistics. ${ }^{11,12}$

Instead of increasing the number of metal particles in a metal-insulator mixture, the insulator-conductor transition can be probed by inducing pressure on an initially nonconducting mixture of metal particles in an insulator, where one or both of the species are deformable. ${ }^{13}$

Many systems, for which the understanding of the physical mechanisms controlling powder compaction is important for the development of new materials, do not contain dc conducting particles. Pharmaceutical tablets are examples of such systems. The compaction properties of drug containing tablets are to a large extent controlled by the properties of the fillers or excipient materials, which usually are nonconducting carbohydrates or celluloses. ${ }^{14}$ The most important excipient material for direct compaction of tablets is microcrystalline cellulose (MCC) derived from wood pulp $\alpha$-cellulose. ${ }^{15}$ The conduction process of MCC at low frequencies is characterized by a quasi-dc conductivity slowly decaying as the frequency is decreased. ${ }^{16}$ This behavior indicates imperfect hopping charge transport. ${ }^{17}$

One of the most commonly used methods to study the 


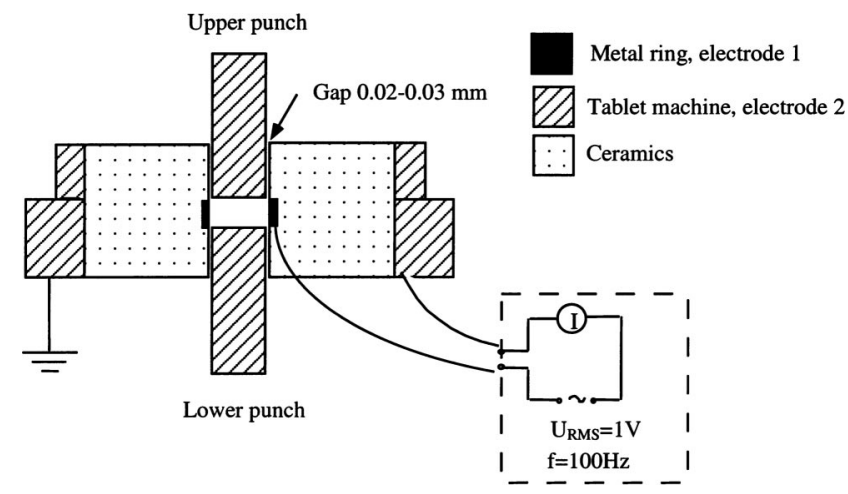

FIG. 1. Schematic cross section of experimental setup with excenterpress in which the MCC is compacted while the conductivity of the sample was recorded. The die cavity has a diameter of $11.3 \mathrm{~mm}$ and is surrounded by a ceramic material. A cylindrical ring, $4 \mathrm{~mm}$ in height, is situated on the die wall and acts as one electrode. The upper and lower punch of the excenterpress are grounded and used as the second electrode.

physics controlling the evolution of the structure that holds up a coherent pharmaceutical tablet, is to measure the tensile strength of the tablet as a function of compaction pressure, ${ }^{18}$ i.e., by studying the mechanical rather than the electrical properties of the tablet.

In this letter, we show that percolation theory developed for analyzing insulator-conductor transitions can be applied to describe the increase in imperfect dc conductivity with densification of porous MCC. Here, the analogue to the twocomponent insulator-conductor system is air-MCC, where the volume fraction of MCC is increased by compression. We also show that this system exactly reproduces the estimated value of percolation threshold and percolation exponent of a 3D random continuum. Finally, we show that the transition from a power-law percolation theory region to a linear effective medium theory region is clearly visible.

Prior to compaction, the MCC (Avicel PH 102, lot 7741, FMC, USA) was conditioned in a desiccator at room temperature over saturated $\mathrm{K}_{2} \mathrm{CO}_{3}$ or $\mathrm{NaCl}$ salt solution ensuring a relative humidity $(\mathrm{RH})$ of 45 and $75 \%$, respectively. The cellulose was taken out of the desiccator and immediately placed in the die of an excenterpress (EK 0, Korsch, Germany) where the material was compacted while the conductivity of the sample was recorded. The experimental setup is displayed in Fig. 1. The die consisted of a cavity with a diameter of $11.3 \mathrm{~mm}$ surrounded by a ceramic material. A cylindrical ring, $4 \mathrm{~mm}$ in height, was situated on the die wall and acted as one electrode. The upper and lower punch of the excenterpress were grounded and used as the second electrode. The excenterpress was operated manually and the tabletting cycle was divided into different positions at which the conductance of the die was recorded. The minimum distance between the lower and the upper punch was set to 5.05 $\mathrm{mm}$. The measuring frequency was chosen as $100 \mathrm{~Hz}$ after measuring the conductance of the MCC filled die in a broad frequency range. At this frequency, the conductance of the MCC, for both moisture contents used in this study, is dominated by imperfect charge transport and is thus rather frequency independent.

Figure 2 shows the conductance of the MCC filled die during compaction for powders equilibrated at $45 \% \mathrm{RH}$. The empty die exhibited a conductance of $55.7 \mathrm{nS}$ independent of

Downloaded 27 Jan 2003 to 130.238.22.132. Redistribution subject

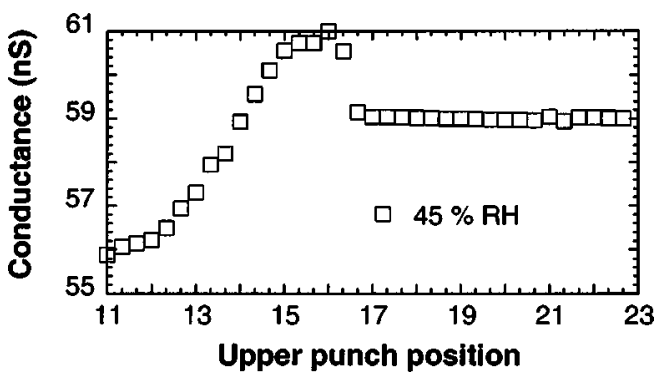

FIG. 2. Conductance vs upper punch position of the MCC filled die during compaction for powders equilibrated at $45 \% \mathrm{RH}$. The data points are averages of approximately 50 measurements.

upper punch position. The data points are averages of approximately 50 measurements. The compaction takes place between upper punch position 11 and 15 and these positions constitute the borders of the interval relevant for the present study. At higher upper punch positions, the upper punch returns to an elevated position no longer contacting the MCC compact and thereafter the lower punch pushes the compact up until it is ejected from the die.

In Fig. 3, the conductance of MCC equilibrated at both 45 and $75 \%$ RH are plotted versus the density of the compacted powder. Here, the conductance of the empty die has been subtracted. The best fits to Eq. (1) for the conductances are also shown. We find that Eq. (1) describes the increase in the conductance for both MCC powders during the densification process with approximately the same parameters $f_{c}$ and $t$ even if the moisture content significantly influences the rate of charge transport, $\sigma_{0}$. The zero porosity density of the MCC under the present study was earlier found to be 1.576 $\mathrm{g} / \mathrm{cm}^{3}{ }^{19}$ Thus, the obtained $f_{c}$ values of 0.238 and 0.249

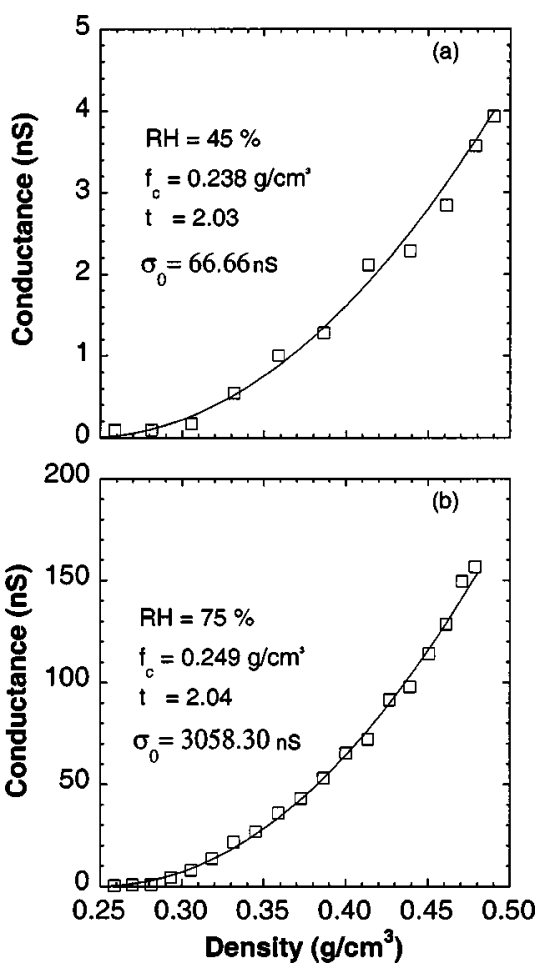

FIG. 3. Conductance of MCC equilibrated at both 45 (a) and 75\% (b) RH vs density of the compacted powder (squares). The conductance of the empty die has been subtracted. The best fits to Eq. (1) for the conductances are also shown (full line), and the fitting parameters are displayed in the insets. 


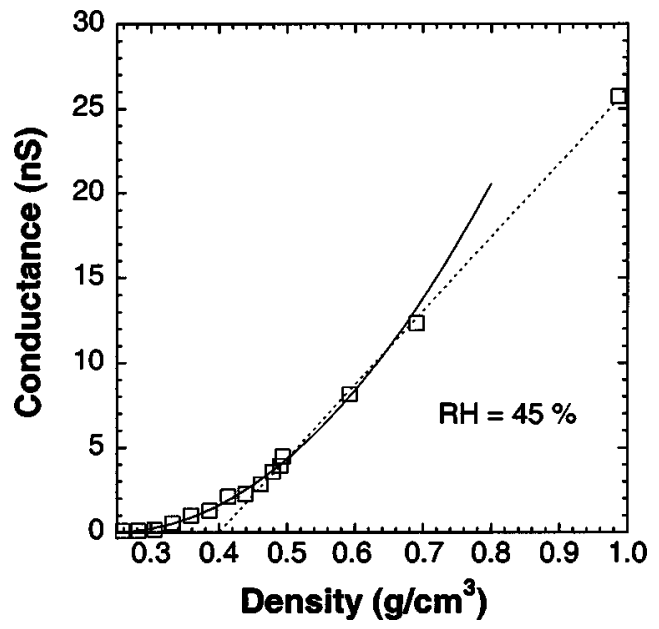

FIG. 4. Conductance of MCC equilibrated at $45 \% \mathrm{RH}$. Values for higher densities have been added to the data presented in Fig. 3(a). The fit to Eq. (1) from Fig. 3(a) (full line) together with a linear fit to the conductance values for the highest densities (dashed line) are also shown.

$\mathrm{g} / \mathrm{cm}^{3}$, respectively, correspond to volume fractions of almost exactly $15 \%$. The increase in $\sigma_{0}$ from a value of $66.7 \mathrm{nS}$ at $45 \% \mathrm{RH}$ to $3058.3 \mathrm{nS}$ at $75 \% \mathrm{RH}$ corresponds very well to the expected increase according to previous ac measurements on MCC compacts for a broad frequency range at different humidities. ${ }^{16}$

To study the conduction behavior of the MCC compacts at higher densities, the die was filled with more material of $45 \%$ RH MCC prior to compaction and the conductance was recorded at the lowest position of the upper punch. In Fig. 4, the conductance values have been added to the original curve shown in Fig. 3(a). Here, we can clearly see that the three data points obtained for the highest densities no longer follow the fit to Eq. (1) (full line). Instead we discover a transition from the Eq. (1) power-law behavior to a linear behavior (dashed line) at densities around $0.5 \mathrm{~g} / \mathrm{cm}^{3}$. This shows that there exists a crossover in the conduction of the porous MCC from a percolation to an effective medium theory behavior at $f \approx 0.3$.

In conclusion, we have reported on the use of percolation theory as a tool in the field of pharmaceutical materials science. Specifically, we have shown that percolation theory, developed for analyzing insulator-conductor transitions, can be applied to describe the increase in imperfect dc conduction during densification of porous pharmaceutical microcrystalline cellulose. We have shown that this system exactly reproduces the estimated value of the percolation threshold and exponent of a 3D random continuum. The transition from a power-law percolation theory region to a linear effective medium theory region is clearly visible from our data.

One of the authors (M.S.) is a Royal Swedish Academy of Sciences (KVA) Fellow and would like to thank the Academy for their support. The Swedish Foundation for Strategic Research (SSF) is gratefully acknowledged for their support to our multidisciplinary research in materials physics and pharmaceutics.

${ }^{1}$ G. Deutscher, A. Kapitulnik, and M. Rappaport, Percolation, Structures, and Processes, edited by G. Deutscher, R. Zallen and J. Adler (Ayalon Offset, Haifa, 1983), p. 207.

${ }^{2}$ Introduction to Percolation Theory, edited by D. Stauffer and A. Aharony (Taylor and Francis, London, 1992).

${ }^{3}$ Applications of Percolation Theory, edited by M. Sahimi (Taylor and Francis, London, 1994).

${ }^{4}$ H. L. Frisch and J. M. Hammersley, J. Soc. Ind. Appl. Math. 11, 894 (1963).

${ }^{5}$ J. P. Clerc, G. Giraud, J. M. Laugier, and J. M. Luck, Adv. Phys. 39, 191 (1990).

${ }^{6}$ S. Kirkpatrick, Rev. Mod. Phys. 45, 574 (1973).

${ }^{7}$ H. Scher and R. Zallen, J. Chem. Phys. 53, 3759 (1970).

${ }^{8}$ D. B. Gingold and C. J. Lobb, Phys. Rev. B 42, 8220 (1990).

${ }^{9}$ G. Deutscher, M. Rappaport, and Z. Ovadyahu, Solid State Commun. 28, 593 (1978).

${ }^{10}$ B. Abeles, Adv. Phys. 24, 407 (1975).

${ }^{11}$ A. Kapitulnik and G. Deutscher, Phys. Rev. Lett. 49, 1444 (1982).

${ }^{12}$ R. F. Voss, R. B. Laibowitz, and E. I. Allessandrini, Phys. Rev. Lett. 49, 1441 (1982)

${ }^{13}$ T. Chelidze and Y. Gueguen, J. Phys. D 31, 2877 (1998).

${ }^{14} \mathrm{G}$. Alderborn, Tablets and Compaction, edited by M. E. Aulton (Churchill Livingstone, New York, 2002).

${ }^{15}$ European Pharmacopoeia (Council of Europe, Strasbourg, 1997).

${ }^{16}$ R. Ek, R. M. Hill, and J. M. Newton, J. Mater. Sci. 32, 4807 (1997).

${ }^{17}$ R. M. Hill and L. A. Dissado, Solid State Ionics 26, 295 (1988).

${ }^{18}$ H. Olsson and C. Nyström, Pharm. Res. 18, 203 (2001).

${ }^{19}$ P. Hedenus, M. Strømme Mattsson, G. A. Niklasson, O. Camber, and R. Ek, Int. J. Pharm. 202, 141 (2000). 